Pacific Journal of Mathematics

REGULAR ELEMENTS IN P.I.-RINGS

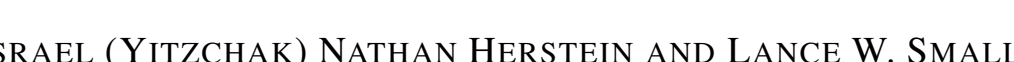




\section{REGULAR ELEMENTS IN P.I.-RINGS}

\section{N. Herstein and Lance W. Small}

It follows from the proof of Posner's theorem that half-regular elements are regular in prime rings satisfying a polynomial identity (prime $P$. I.-rings). In this paper we extend these results to semi-prime rings and present counterexamples to several avenues of further generalization.

Throughout this paper all rings will be algebras over a commutative ring. We further assume that the polynomial identities which occur have at least one invertible coefficient. If $T$ is a subset of a ring $R$ then $l(T)(r(T))$ will denote the left (right) annihilator of $T$. The word "ideal" will mean two-sided ideal. Finally, we recall that if $R$ is semi-prime and if $U$ is an ideal of $R$ then $l(U)=r(U)$. In this case we write $l(U)$, unambiguously, as $\operatorname{Ann}(U)$.

2. We begin with a mild generalization of a result due to Amitsur [1].

LEMma 1. Let $R$ be a ring such that $R a$ satisfies a polynomial identity; then, if $l(a)=0, R a$ contains a nonzero ideal of $R$.

Proof. Among the left ideals $R a^{i}$ suppose that $R a^{k}$ satisfies an identity of lowest degree. We may assume that this identity is multilinear and has form

$$
q\left(x_{1} \cdots, x_{n}\right)=q_{1}\left(x_{1}, \cdots, x_{n-1}\right) x_{n}+q_{2}\left(x_{1}, \cdots, x_{n}\right)
$$

where $q_{1}$ is of lower degree than $q$ and where $x_{n}$ does not occur as the last variable of any monomial of $q_{2}$. Substitute $r_{j} a^{2 k}$ for $x_{j}$ for $j=1, \cdots, n-1$ and $r_{n} a^{k}$ for $x_{n}$, where $r_{1}, \cdots, r_{n}$ are arbitrary elements of $R$, in $q\left(x_{1}, \cdots, x_{n}\right)$. Since $R a^{2 k} \subset R a^{k}, R a^{2 k}$ satisfies $q$ and, by our choice of $k$, no identity of lower degree. Therefore there exist $r_{1}, \cdots, r_{n-1}$ in $R$ such that $q_{1}\left(r_{1} a^{2 k}, \cdots, r_{n-1} a^{2 k}\right) \neq 0$. Freeding this into our identity $q$ we obtain

$$
q_{1}\left(r_{1} a^{2 k}, \cdots, r_{n-1} a^{2 k}\right) r_{n} a^{k}=-q_{2}\left(r_{1} a^{2 k}, \cdots, r_{n-1} a^{2 k}, r_{n} a^{k}\right)
$$

which is contained in $R \alpha^{2 k}$ from the form of $q_{2}$. Since $l(a)=0$ this yields $q_{1}\left(r_{1} a^{2 k}, \cdots, r_{n-1} a^{2 k}\right) r_{n} \in R a^{k}$. In short, $q_{1}\left(r_{1} a^{2 k}, \cdots, r_{n-1} a^{2 k}\right) R \subset R a^{k}$, hence the nonzero ideal $R q_{1}\left(r_{1} a^{2 k}, \cdots, r_{n-1} a^{2 k}\right) R$ is contained in $R a^{k}$, and so, in $R a$. This proves the result.

The plan now is to study $R a$ by looking at the ideals of $R$ contained in it. The crucial step is 
THEOREM 2. Suppose that $R$ is a semi-prime ring; if $a \in R$ is such that $l(a)=0$ and $R a$ satisfies a polynomial identity then $R a$ contains an ideal of $R$ whose annihilator is zero.

Proof. Let $U$ be the sum of the ideals of $R$ which are contained in $R a$. We claim that $\operatorname{Ann}(U)=0$. If not, let $W=\operatorname{Ann}(U) \neq 0$, and $V=\operatorname{Ann}(W)$. Pass to the ring $\bar{R}=R / V$. If $\bar{x} \bar{a}=0$ in $\bar{R}$ then $x a \in V$ hence $W x a=0$; since $l(a)=0$ this gives $W x=0$, and so, $x \in V, \bar{x}=0$. Thus $l(\bar{a})=0$. Clearly $\bar{R} \bar{a}$ satisfies a polynomial identity. Therefore $\bar{R} \bar{a}$ contains a nonzero ideal $\bar{T}$ of $\bar{R}$; the inverse image $T$ of $\bar{T}$ thus lies in $R a+V$. Since $\bar{T} \neq 0, T \not \subset V$ therefore $0 \neq W T \subset R a+W V$. But $W V=0$. Consequently $W T$ is a nonzero ideal of $R$ lying in $R a$. As such, it must be contained in $U$. But $W U=0$, so $(W T)^{2} \subset W^{2} T=0$. Thus semi-primeness of $R$ then forces the contradiction $W T=0$. With this, the theorem is proved.

From Theorem 2 many good things flow.

THEOREM 3. Suppose that $R$ is a semi-prime P.I.-ring. If $a \in R$ satisfies $l(a)=0$ then

1. $r(a)=0$

2. $R a$ is essential.

Proof. 1. Let $U$ be the ideal in $R a$ of Theorem 2. If $a x=0$ then $U x=0$, which is not possible unless $x=0$. Thus $r(a)=0$.

2. If $I$ is a nonzero left ideal then $0 \neq U I \subset U \cap I \subset R a \cap I$.

A ring $R$ is said to be von Neumann finite if for $x, y \in R, x y=1$ implies $y x=1$. If $R_{n}$ is $\mathrm{v}$. $\mathrm{N}$. finite for all $n$, we call $R N$-finite.

Corollary. A P. I.-ring is $N$-finite.

Proof. The result follows easily from the following two observations:

1. if $R$ is a P. I.-ring then $R_{n}$ is a P. I.-ring [3].

2. $R$ is $\mathrm{v}$. N. finite if and only if $R / J(R)$ is, where $J(R)$ is the Jacobson radical of $R$.

Hence we can reduce to the semi-simple (and so, semi-prime) case. If $x y=1$ then $l(x)=0$ where, by Theorem $3, r(x)=0$. Since $x(1-y x)=0$ we get $y x=1$.

Theorem 2 also tells us something about the nature of the identities satisfied by $R$ and $R a$.

THEOREM 4. If $R$ is a semi-prime ring and if $a \in R$ satisfies $l(a)=0$ then $R$ satisfies any polynomial identity satisfied by $R a$. 
Proof. The argument follows one by Goldie [2]. Since $R$ is semiprime, $0=\cap P_{\alpha}$ where $P_{\alpha}$ are prime ideals. Let $U \subset R a$ be an ideal of $R$ such that $\operatorname{Ann}(U)=0$. Now $U \not \subset P_{\beta}$ for some prime ideal $P_{\beta}$. Divide the prime ideals of $R$ into two parts: those which contain $U$ and those which do not. The intersection of the primes in the first part contains $U$ and is annihilated by the intersection of the primes in the second part. But $\operatorname{Ann}(U)=0$, so this latter intersection must be 0 . Hence $0=\cap P_{r}$ where the $P_{\gamma}$ are prime ideals and $U \not \subset P_{\gamma}$ for each $\gamma$. We find, then, that $R_{r}=R / P_{r}$ has a nonzero ideal $\left(U+P_{r}\right) / P_{r}$ which satisfies an identity. Since $R_{r}$ is prime, it satisfies the same identity as $\left(U+P_{\gamma}\right) / P_{\gamma}$ [1]. To finish up, we note that $R$ is a subdirect sum of the $R_{r}$, hence satisfies any identity of $U$, therefore any identity of $R a$.

3. In this section we present several counter-examples to possible generalizations of the results in $\S 2$. We begin with examples to show that "semi-prime" is needed in Theorem 3.

Let $F$ be a field and $F[x]$ the polynomial ring in $x$ over $F$. Form the ring $S^{(1)}=\left(\begin{array}{cc}F[x] & F \\ 0 & F\end{array}\right)$, where $F[x]$ acts on $F$ in the usual way (identifying $F=F[x] /(x)$ as an $F[x]$-module). $\quad \mathbf{S}^{(1)}$ satisfies the identity $(a b-b a)^{2}=0$. It is easy to see that $l\left(\begin{array}{ll}x & 0 \\ 0 & 1\end{array}\right)=0$, but $r\left(\begin{array}{cc}x & 0 \\ 0 & 1\end{array}\right) \neq 0$.

Now form the ring $S^{(2)}=\left(\begin{array}{cc}F[x] & F[x] \\ 0 & F\end{array}\right)$ with the obvious actions on $F[x]$. $S^{(2)}$ satisfies the same identity as $S^{(1)}$. The element $\left(\begin{array}{cc}x & 0 \\ 0 & 1\end{array}\right)$ is regular in $S^{(2)} \operatorname{but}\left(\begin{array}{ll}x & 0 \\ 0 & 1\end{array}\right) S^{(2)} \cap\left(\begin{array}{ll}0 & F \\ 0 & 0\end{array}\right)=0$-that is, the right ideal $\left(\begin{array}{ll}x & 0 \\ 0 & 1\end{array}\right) S^{(2)}$ is not essential. We pause to note that this implies that $S^{(2)}$ does not satisfy the right Ore condition. Yet $S^{(2)}$ possesses a ring of left quotients which even is Artinian.

We conclude this section with a simple example of a right Noetherian ring which lacks a right ring of quotients. Let $R$ be any commutative Noetherian ring with the following property: there exists an element $a \in R$ which is not regular but its image, $\bar{a}$, is regular in $\bar{R}=R / N$ where $N$ is the nil radical of $R$. (An example of such is $\frac{F[x, y]}{\left(x^{2}, x y\right)}$ where $a=y+\left(x^{2}, x y\right)$.) Our example is $S^{(2)}=\left(\begin{array}{cc}\bar{R} & \bar{R} \\ 0 & R\end{array}\right)$.

The element $\left(\begin{array}{ll}\bar{a} & 0 \\ 0 & 1\end{array}\right)$ is quickly seen to be regular in $S^{(3)}$. If the right Ore condition were valid we would have an equation

$$
\left(\begin{array}{ll}
\bar{a} & 0 \\
0 & 1
\end{array}\right)\left(\begin{array}{ll}
\bar{d} & \bar{c} \\
0 & b
\end{array}\right)=\left(\begin{array}{ll}
0 & \overline{1} \\
0 & 0
\end{array}\right)\left(\begin{array}{ll}
\bar{r} & \bar{s} \\
0 & t
\end{array}\right)
$$


where $\left(\begin{array}{cc}\bar{r} & \bar{s} \\ 0 & t\end{array}\right)$ was regular. This forces $t$ to be regular in $R$. Writing the relations out explicitly, we have $\bar{a} \bar{c}=\bar{t}$, which means that $a c=t+n$ where $n \in N$. But $t$ is regular, hence $t+n$ is and so $a c$ is regular. This contradicts our choice of $a$.

4. To finish up, we present a result on the rank of free modules over P. I.-rings which, for commutative rings, is a well-known theorem on homogeneous systems of linear equations. The proof we give may be of additional interest in that we cannot, of course, use determinants.

Denote by ${ }_{R} R^{(n)}$ the external direct sum of $n$ copies of ${ }_{R} R$, that is, the free module on $n$ basis elements.

TheOREM 5. If $R$ is a P. I.-ring, then $R^{(n)} \subset R^{(m)}$ implies $n \leqq m$.

Proof. Suppose that $n>m$. First note that this forces $R^{(t)} \subset R^{(m)}$ for arbitrary to To see this, write $R^{(n)}=R^{(m)} \oplus R^{(n-m)}$. We can find a copy of $R^{(n)}$ in the first summand, so $R^{(n)} \oplus R^{(n-m)} \subset R^{(m)}$. We now repeat the process on the "new" $R^{(n)}$. In particular, we obtain $R^{(2 m)} \subset R^{(m)}$. This means that $R^{(m)}$ contains a set, $\alpha_{1}, \cdots, \alpha_{2 m}$, of $2 m$ linearly independent elements. We can consider the $\alpha$ 's as $1 \times m$ row vectors and form the $m \times m$ matrices $X$ and $Y$ where the rows of $X$ are $\alpha_{1}, \cdots, \alpha_{m}$ and those of $Y$ are $\alpha_{m+1}, \cdots, \alpha_{2 m}$. In $R_{m}$ it is immediate that $l(X)=0$ and $l(Y)=0$ since $\alpha_{1}, \cdots, \alpha_{2 m}$ are independent. But $R_{m}$ is a P. I.-ring, so by Lemma $1 R_{m} X$ contains a nonzero ideal $U$. Now, since $l(Y)=0, U R_{m} Y \neq 0$ and is contained in $R_{m} X$. This yields nonzero matrices $A$ and $B$ such that $A X=B Y$. Writing this out explicitly gives a dependence relation among the $\alpha$ 's, a contradiction. The proof is complete.

\section{REFERENCES}

1. S. A. Amitsur, Prime rings satisfying polynomial identities with arbitrary coefficients, Proc. London Math. Soc. (3) 17 (1967), 470-486.

2. A. W. Goldie, A note on prime rings with polynomial identities, London Math. Soc. (2), 1 (1969), 606-608.

3. C. Procesi and L. Small, Endomorphism rings of modules over P. I.-algebras, Math. Zeit. 106 (1968), 178-180.

Received September 8, 1970. The research of the second named author was supported by an NSF grant at the University of Southern California.

UNIVERSity OF CHICAgo,

University of SOUthern California

and

University of California, San Diego. 


\section{PACIFIC JOURNAL OF MATHEMATICS}

\section{EDITORS}

H. SAMELson

Stanford University

Stanford, California 94305

R. R. PHelPS

University of Washington

Seattle, Washington 98105
J. Dugundu

Department of Mathematics

University of Southern Californle

Los Angeles, California 9.0007

RICHARD ARENS

University of California

Los Angeles, California 9.0024

\section{ASSOCIATE EDITORS}
E. F. BECKENBACH
B. H. NeumanN
F. WOLE
K. Yoshida

\section{SUPPORTING INSTITUTIONS}

UNIVERSITY OF BRITISH COLUMBIA

CALIFORNIA INSTITUTE OF TECHNOLOGY

UNIVERSITY OF CALIFORNIA

MONTANA STATE UNIVERSITY

UNIVERSITY OF NEVADA

NEW MEXICO STATE UNIVERSITY

OREGON STATE UNIVERSITY

UNIVERSITY OF OREGON

OSAKA UNIVERSITY

UNIVERSITY OF SOUTHERN CALIFORNIA

\author{
STANFORD UNIVERSITY \\ UNIVERSITY OF TOKYO \\ UNIVERSITY OF UTAH \\ WASHINGTON STATE UNIVERSITY \\ UNIVERSITY OF WASHINGTON \\ AMERICAN MATHEMATICAL SOCIETY \\ CHEVRON RESEARCH CORPORATION \\ NAVAL WEAPONS CENTER
}

The Supporting Institutions listed above contribute to the cost of publication of this Journal, but they are not owners or publishers and have no responsibility for its content or policies.

Mathematical papers intended for publication in the Pacific Journal of Mathematics should be in typed form or offset-reproduced, (not dittoed), double spaced with large margins. Underline Greek letters in red, German in green, and script in blue. The first paragraph or two must be capable of being used separately as a synopsis of the entire paper. The editorial "we" must not be used in the synopsis, and items of the bibliography should not be cited there unless absolutely necessary, in which case they must be identified by author and Journal, rather than by item number. Manuscripts, in duplicate if possible, may be sent to any one of the four editors. Please classify according to the scheme of Math. Rev. Index to Vol. 39. All other communications to the editors should be addressed to the managing editor, Richard Arens, University of California, Los Angeles, California, 90024.

50 reprints are provided free for each article; additional copies may be obtained at cost in multiples of 50 .

The Pacific Journal of Mathematics is published monthly. Effective with Volume 16 the price per volume (3 numbers) is $\$ 8.00$; single issues, $\$ 3.00$. Special price for current issues to individual faculty members of supporting institutions and to individual members of the American Mathematical Society: $\$ 4.00$ per volume; single issues $\$ 1.50$. Back numbers are available.

Subscriptions, orders for back numbers, and changes of address should be sent to Pacific Journal of Mathematics, 103 Highland Boulevard, Berkeley, California, 94708.

PUBLISHED BY PACIFIC JOURNAL OF MATHEMATICS, A NON-PROFIT CORPORATION

Printed at Kokusai Bunken Insatsusha (International Academic Printing Co., Ltd.), 7-17, Fujimi 2-chome, Chiyoda-ku, Tokyo, Japan. 


\section{Pacific Journal of Mathematics}

\section{Vol. 36, No. 2 December, 1971}

George E. Andrews, On a partition problem of H. L. Alder ............ 279

Thomas Craig Brown, An interesting combinatorial method in the theory of locally finite semigroups .......................... 285

Yuen-Kwok Chan, A constructive proof of Sard's theorem ............. 291

Charles Vernon Coffman, Spectral theory of monotone Hammerstein

operators...................................... 303

Edward Dewey Davis, Regular sequences and minimal bases .......... 323

Israel (Yitzchak) Nathan Herstein and Lance W. Small, Regular elements in

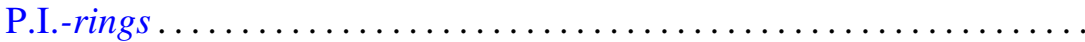

Marcel Herzog, Intersections of nilpotent Hall subgroups ..............

W. N. Hudson, Volterra transformations of the Wiener measure on the space

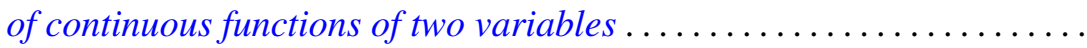

J. H. V. Hunt, An n-arc theorem for Peano spaces ................ 351

Arnold Joseph Insel, A decomposition theorem for topological group

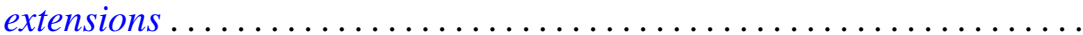

Caulton Lee Irwin, Inverting operators for singular boundary value

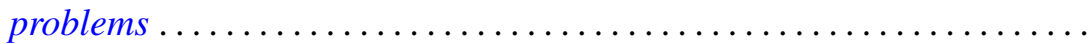

Abraham A. Klein, Matrix rings of finite degree of nilpotency ............ 387

Wei-Eihn Kuan, On the hyperplane section through a rational point of an algebraic variety...

John Hathway Lindsey, II, On a six-dimensional projective representation of $\mathrm{PSU}_{4}(3)$

Jorge Martinez, Approximation by archimedean lattice cones ...

J. F. McClendon, On stable fiber space obstructions .........

Mitsuru Nakai and Leo Sario, Behavior of Green lines at the Kuramochi boundary of a Riemann surface ....................

Donald Steven Passman, Linear identities in group rings. I. .

Donald Steven Passman, Linear identities in group rings. II ...

David S. Promislow, The Kakutani theorem for tensor products of

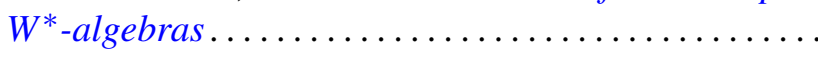

Richard Lewis Roth, On the conjugating representation of a finite group

Bert Alan Taylor, On weighted polynomial approximation of entire functions...

William Charles Waterhouse, Divisor classes in pseudo Galois

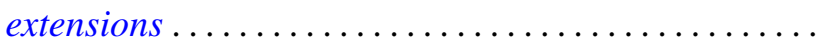

Chi Song Wong, Subadditive functions ...

Ta-Sun $\mathrm{Wu}$, A note on the minimality of certain bitransformation groups 